\title{
Caddo Ceramic Vessel Database from Sites in Texas, Louisiana, Oklahoma, and Arkansas
}

Timothy K. Perttula

Heritage Research Center, Stephen F. Austin State University

Follow this and additional works at: https://scholarworks.sfasu.edu/ita

Part of the American Material Culture Commons, Archaeological Anthropology Commons, Environmental Studies Commons, Other American Studies Commons, Other Arts and Humanities Commons, Other History of Art, Architecture, and Archaeology Commons, and the United States History Commons

Tell us how this article helped you.

This Article is brought to you for free and open access by the Center for Regional Heritage Research at SFA ScholarWorks. It has been accepted for inclusion in Index of Texas Archaeology: Open Access Gray Literature from the Lone Star State by an authorized editor of SFA ScholarWorks. For more information, please contact cdsscholarworks@sfasu.edu. 
Caddo Ceramic Vessel Database from Sites in Texas, Louisiana, Oklahoma, and Arkansas

\section{Creative Commons License}

\section{(c) (1) (9)}

This work is licensed under a Creative Commons Attribution-NonCommercial 4.0 International License 


\title{
Caddo Ceramic Vessel Database from Sites in Texas, Louisiana, Oklahoma, and Arkansas
}

\author{
Timothy K. Perttula
}

This compilation of more than 9310 ancestral Caddo ceramic vessels is from 356 sites and collections primarily in Texas (i.e., about 87 percent of the vessels and 88 percent of the sites and collections), although there are significant collections in this compilation from sites in Arkansas, Louisiana, and Oklahoma. The ceramic vessel database has been compiled from available published and unpublished articles, monographs, and technical reports, as well as ceramic vessel inventory forms (when available) on file at the Texas Archeological Research Laboratory.

Many of these vessels have been documented over the last 10 years using a standard analysis protocol. Nevertheless, it is expected that not all known vessel collections have been included in this database from any one state in the southern Caddo area (most assuredly not from southwestern Arkansas, where McKinnon [2016:31] had noted that he has a GIS database of 4199 vessels from 187 sites that are mostly in Arkansas). If and when other vessel collections not presently included herein are identified, every effort will be made in the future to add them into the ceramic vessel database so that it is as comprehensive a listing of vessels as possible.

Each individual vessel entry includes the following: (1) site trinomial, if known, or collection name (along with a county location, if no trinomial is available); (2) vessel and/or burial number, or other accession/catalog information; (3) vessel form; (4) temper, if known; (5) type, if known; (6) variety, if known; (7) decorative method or decorative elements for vessels of unknown type-variety, plus other pertinent information on vessels; and (8) estimated age of the sites that have vessels from burial and/or habitation features.

The majority of the site and collection vessel assemblages in the data base-current as of August 14, 2016-have less than 30 ceramic vessels (Tables 1-4). However, there are several sites with substantial vessel assemblages in each of the four states within the southern Caddo area, including, for Texas: Tuck Carpenter (41CP5, n=468 vessels), Johns (41CP12, n=257 vessels), H. R. Taylor (41HS3, n=427 vessels), Holdeman (41RR11, n=259 vessels), Sam Kaufman (41RR16, n=414 vessels), Henry Spencer 41UR315, $\mathrm{n}=236$ vessels); the Belcher site (16CD13, $\mathrm{n}=190$ vessels) in Louisiana; the Roden site (34MC215, $\mathrm{n}=110$ vessels) in Oklahoma; and the Battle (3LA1, $\mathrm{n}=163$ vessels) and Crenshaw (3MI6, $\mathrm{n}=252$ vessels) sites in southwest Arkansas. Ceramic vessels dating from all periods in Caddo native history are present in the database (i.e., Formative Caddo, ca. A.D. 800-1000; Early Caddo, ca. A.D. 10001200; Middle Caddo, ca. A.D. 1200-1400; Late Caddo, ca. A.D. 1400-1680; and Historic Caddo, ca. A.D. 1680-1850s), but ceramic vessels dating to the Late Caddo period are predominant in each of the vessel assemblages from the four states. In particular, these are vessels from assemblages belonging to the Belcher phase, Frankston phase, McCurtain phase, Salt Lick, Texarkana, and Titus phases that are found along the Red River and in the Neches-Angelina, and Big Cypress-Sabine drainage basins in East Texas (Figure 1). 


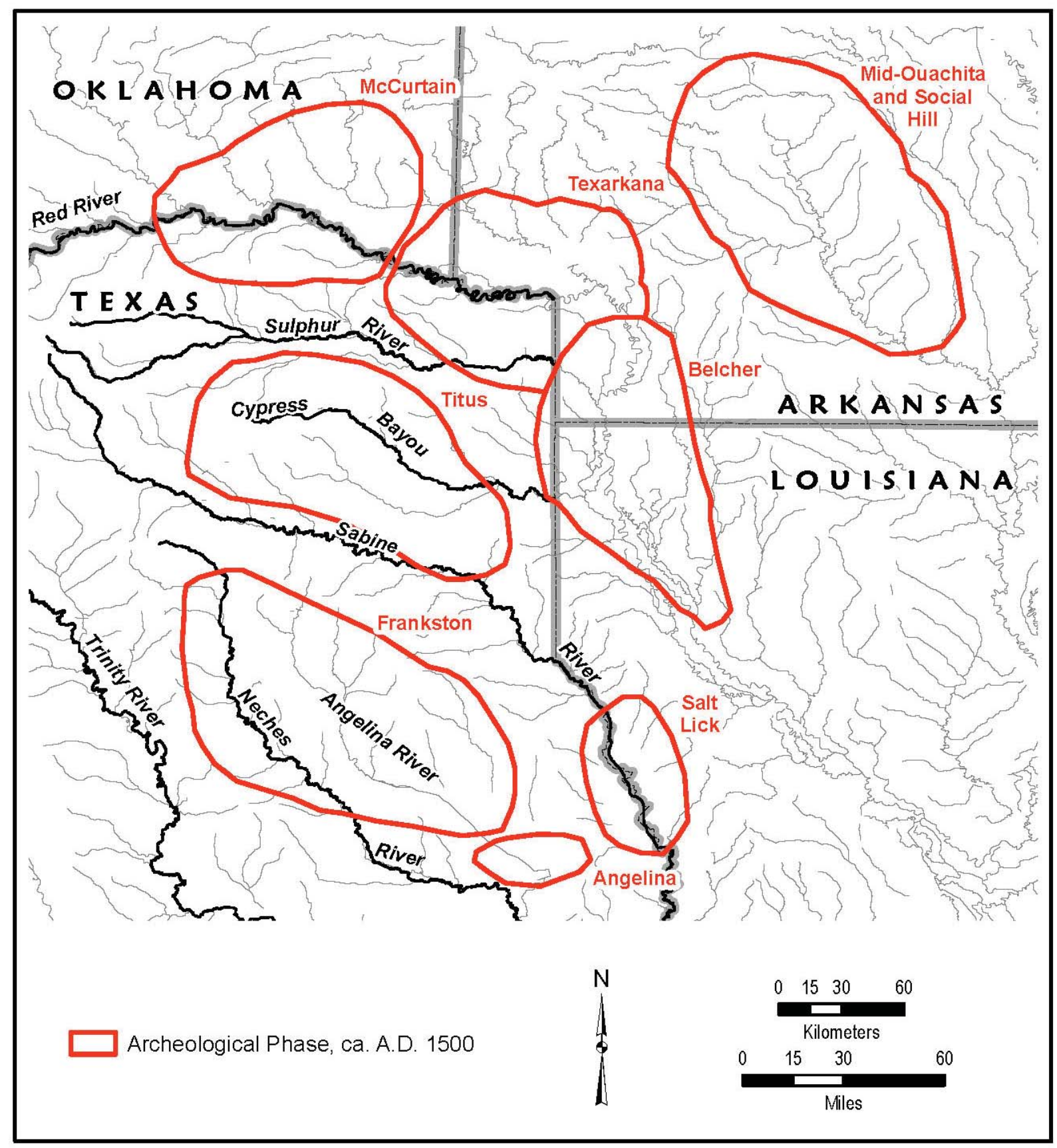

Figure 1. Late Caddo period phases in southwest Arkansas, northwest Louisiana, southeast Oklahoma, and East Texas. Figure prepared by Sandra Hannum. 
Table 1. East Texas sites/collections with Caddo ceramic vessels.

\begin{tabular}{|c|c|}
\hline Site (with trinomial) or Collection (by County) & No. of vessels in database \\
\hline 41AG66 & 3 \\
\hline $41 \mathrm{AN} 1$ & 33 \\
\hline 41AN2 & 17 \\
\hline $41 \mathrm{AN} 13$ & 13 \\
\hline $41 \mathrm{AN} 14$ & 14 \\
\hline $41 \mathrm{AN} 16$ & 1 \\
\hline 41AN18 & 2 \\
\hline $41 \mathrm{AN} 21$ & 1 \\
\hline $41 \mathrm{AN} 26$ & 25 \\
\hline 41AN32 & 23 \\
\hline $41 \mathrm{AN} 34$ & 19 \\
\hline 41AN38 & 31 \\
\hline 41AN39 & 9 \\
\hline 41AN44 & 12 \\
\hline $41 \mathrm{AN} 48$ & 3 \\
\hline $41 \mathrm{AN} 53$ & 4 \\
\hline 41AN54 & 15 \\
\hline 41AN56 & 1 \\
\hline 41AN57 & 3 \\
\hline $41 \mathrm{AN} 67$ & 1 \\
\hline 41AN184 & 4 \\
\hline 41AN201/41HE7 & 8 \\
\hline McSpadden & 3 \\
\hline NMNH & 4 \\
\hline 41BW1 & 1 \\
\hline $41 \mathrm{BW} 2$ & 12 \\
\hline $41 \mathrm{BW} 3$ & 69 \\
\hline 41BW4 & 149 \\
\hline 41BW5 & 13 \\
\hline 41BW8 & 29 \\
\hline 41BW14 & 11 \\
\hline 41BW33 & 6 \\
\hline 41BW65 & 1 \\
\hline BW119 & 2 \\
\hline BW171 & 1 \\
\hline BW512 & 4 \\
\hline Winston Md. (Bowie Co.) & 4 \\
\hline Boyce Smith Collection (Bowie Co.) & 1 \\
\hline $41 \mathrm{CE} 4$ & 24 \\
\hline 41CE6 & 8 \\
\hline $41 \mathrm{CE} 12$ & 55 \\
\hline 41CE14-Cemetery 1 & 106 \\
\hline 41CE14-Cemetery 2 & 12 \\
\hline
\end{tabular}


Table 1. East Texas sites/collections with Caddo ceramic vessels, cont.

\begin{tabular}{|c|c|}
\hline Site (with trinomial) or Collection (by County) & No. of vessels in database \\
\hline 41CE15 & 3 \\
\hline 41CE17 & 35 \\
\hline 41CE19 & 47 \\
\hline 41CE20 & 1 \\
\hline $41 \mathrm{CE} 23$ & 8 \\
\hline 41CE25 & 27 \\
\hline 41CE43 & 1 \\
\hline $41 \mathrm{CE} 421$ & 1 \\
\hline Mud Creek & 4 \\
\hline H. C. Slider & 5 \\
\hline Upton & 3 \\
\hline Tidwell & 1 \\
\hline $\mathrm{NMNH}$ & 11 \\
\hline Boyce Smith Collection (Cherokee Co.) & 10 \\
\hline $41 \mathrm{CP} 2$ & 52 \\
\hline $41 \mathrm{CP} 3$ & 42 \\
\hline $41 \mathrm{CP} 5$ & 468 \\
\hline $41 \mathrm{CP} 10$ & 34 \\
\hline $41 \mathrm{CP} 12$ & 257 \\
\hline $41 \mathrm{CP} 17$ & 31 \\
\hline $41 \mathrm{CP} 20$ & 38 \\
\hline $41 \mathrm{CP} 25$ & 4 \\
\hline $41 \mathrm{CP} 71$ & 91 \\
\hline $41 \mathrm{CP} 76$ & 3 \\
\hline $41 \mathrm{CP} 78$ & 14 \\
\hline $41 \mathrm{CP} 183$ & 1 \\
\hline $41 \mathrm{CP} 244$ & 37 \\
\hline $41 \mathrm{CP} 304$ & 154 \\
\hline $41 \mathrm{CP} 408$ & 19 \\
\hline Lineburger & 2 \\
\hline Boyd Farm & 1 \\
\hline Hilton Farm & 10 \\
\hline Boyce Smith Collection (Camp Co.) & 7 \\
\hline $41 \mathrm{CS} 1$ & 1 \\
\hline $41 \mathrm{CS} 5$ & 9 \\
\hline $41 \mathrm{CS} 7$ & 9 \\
\hline $41 \mathrm{CS} 8$ & 5 \\
\hline $41 \mathrm{CS} 14$ & 16 \\
\hline $41 \mathrm{CS} 23$ & 69 \\
\hline $41 \mathrm{CS} 25$ & 83 \\
\hline $41 \mathrm{CS} 26$ & 17 \\
\hline $41 \mathrm{CS} 37$ & 5 \\
\hline $41 \mathrm{CS} 132$ & 2 \\
\hline $41 \mathrm{CS} 133$ & 4 \\
\hline
\end{tabular}


Table 1. East Texas sites/collections with Caddo ceramic vessels, cont.

\begin{tabular}{|c|c|}
\hline Site (with trinomial) or Collection (by County) & No. of vessels in database \\
\hline $41 \mathrm{FK} 1$ & 22 \\
\hline 41FK2 & 52 \\
\hline 41FK3 & 13 \\
\hline 41FK4 & 84 \\
\hline $41 \mathrm{FK} 5$ & 12 \\
\hline 41FK32 & 11 \\
\hline 41FK97 & 19 \\
\hline 41FK107 & 2 \\
\hline Good Hope & 17 \\
\hline 41FN12 & 1 \\
\hline 41GG3 & 6 \\
\hline 41GG5 & 25 \\
\hline $41 \mathrm{GG} 50$ & 28 \\
\hline Glade Creek & 1 \\
\hline Hyte & 27 \\
\hline 41HE4/55 & 4 \\
\hline 41HE75 & 9 \\
\hline 41HE78 & 1 \\
\hline $41 \mathrm{HE} 82$ & 1 \\
\hline $41 \mathrm{HE} 114$ & 21 \\
\hline NMNH & 4 \\
\hline 41HP1 & 46 \\
\hline 41HP2 & 4 \\
\hline 41HP15 & 5 \\
\hline 41HP26 & 2 \\
\hline $41 \mathrm{HP} 237$ & 15 \\
\hline 41HР239 & 3 \\
\hline $41 \mathrm{HS} 1$ & 5 \\
\hline $41 \mathrm{HS} 3$ & 427 \\
\hline $41 \mathrm{HS} 13$ & 22 \\
\hline 41HS14 & 3 \\
\hline 41HS15 & 150 \\
\hline 41HS74 & 23 \\
\hline 41HS235 & 84 \\
\hline 41HS253 & 28 \\
\hline 41HS261 & 32 \\
\hline 41HS262 & 1 \\
\hline $41 \mathrm{HS} 269$ & 14 \\
\hline 41HS718 & 1 \\
\hline $41 \mathrm{HS} 825$ & 105 \\
\hline Darco Coal Mine & 4 \\
\hline
\end{tabular}


Table 1. East Texas sites/collections with Caddo ceramic vessels, cont.

\begin{tabular}{|c|c|}
\hline Site (with trinomial) or Collection (by County) & No. of vessels in database \\
\hline Eli Fields & 21 \\
\hline Loftis & 2 \\
\hline Marshall Red Gully & 3 \\
\hline Martin & 2 \\
\hline P. Smith & 1 \\
\hline Boyce Smith Collection (Harrison Co.) & 15 \\
\hline 41LR1 & 21 \\
\hline 41LR2 & 80 \\
\hline 41LR39 & 4 \\
\hline 41MR6 & 10 \\
\hline $41 \mathrm{MR} 12$ & 48 \\
\hline $41 \mathrm{MR} 63$ & 12 \\
\hline Boyce Smith Collection (Marion Co.) & 7 \\
\hline $41 \mathrm{MX} 1$ & 33 \\
\hline $41 \mathrm{MX} 2$ & 83 \\
\hline $41 \mathrm{MX} 5$ & 6 \\
\hline $41 \mathrm{MX} 6$ & 6 \\
\hline $41 \mathrm{MX} 9$ & 3 \\
\hline $41 \mathrm{MX} 65$ & 38 \\
\hline Harold Nix & 51 \\
\hline Sulphur River & 7 \\
\hline 41NA3 & 1 \\
\hline 41NA22 & 1 \\
\hline 41NA27 & 13 \\
\hline 41NA49 & 49 \\
\hline 41NA113 & 2 \\
\hline 41NA202 & 5 \\
\hline 41NA231 & 5 \\
\hline 41NA285 & 1 \\
\hline Indian $\mathrm{Cr}$. & 1 \\
\hline 41PN48 & 4 \\
\hline Beckville & 1 \\
\hline 41RA13 & 45 \\
\hline 41RK2 & 5 \\
\hline 41RK3 & 14 \\
\hline 41RK79 & 4 \\
\hline 41RK80 & 2 \\
\hline 41RK132 & 4 \\
\hline 41RK170 & 6 \\
\hline
\end{tabular}


Table 1. East Texas sites/collections with Caddo ceramic vessels, cont.

\begin{tabular}{|c|c|}
\hline Site (with trinomial) or Collection (by County) & No. of vessels in database \\
\hline $41 \mathrm{RK} 214$ & 11 \\
\hline 41RK602 & 8 \\
\hline Bead & 5 \\
\hline Morwell & 2 \\
\hline J. T. Hughes Collection & 10 \\
\hline 41RR2 & 13 \\
\hline 41RR3 & 9 \\
\hline 41RR7 & 1 \\
\hline 41RR8 & 13 \\
\hline 41RR9 & 2 \\
\hline 41RR11 & 259 \\
\hline 41RR16 & 414 \\
\hline 41RR41 & 43 \\
\hline 41RR77 & 192 \\
\hline Jim Clark (Red River Co.) & 1 \\
\hline Shawnee Town (Red River Co.) & 1 \\
\hline Boyce Smith Collection (Red River Co.) & 14 \\
\hline 41SA89 & 1 \\
\hline 41SA94 & 5 \\
\hline 41SA116 & 2 \\
\hline $41 \mathrm{SA} 123$ & 1 \\
\hline $41 \mathrm{SB} 35$ & 2 \\
\hline $41 \mathrm{SB} 50$ & 12 \\
\hline 41SB71 & 1 \\
\hline 41SM2 & 2 \\
\hline 41SM55 & 4 \\
\hline 41SM56 & 7 \\
\hline 41SM73 & 15 \\
\hline 41SM77 & 31 \\
\hline 41SM90 & 8 \\
\hline 41SM93 & 12 \\
\hline 41SM150 & 15 \\
\hline 41SM151 & 3 \\
\hline 41SM158 & 2 \\
\hline 41SM193 & 30 \\
\hline 41SM197 & 6 \\
\hline 41SM198 & 7 \\
\hline 41SM223 & 2 \\
\hline 41SM246 & 2 \\
\hline 41SM247 & 6 \\
\hline 41SM248 & 4 \\
\hline 41SM249 & 20 \\
\hline
\end{tabular}


Table 1. East Texas sites/collections with Caddo ceramic vessels, cont.

\begin{tabular}{|c|c|}
\hline Site (with trinomial) or Collection (by County) & No. of vessels in database \\
\hline 41SM262 & 2 \\
\hline 41SM268 & 1 \\
\hline 41SM282 & 2 \\
\hline 41SM290 & 21 \\
\hline 41SM294 & 1 \\
\hline 41SM355 & 3 \\
\hline Saline Creek & 5 \\
\hline Indian Creek & 1 \\
\hline Byars Ranch & 1 \\
\hline Smith Co. & 12 \\
\hline TJC (Smith Co.) & 9 \\
\hline $41 \mathrm{SY} 24$ & 4 \\
\hline $41 \mathrm{SY} 25$ & 3 \\
\hline $41 \mathrm{SY} 27$ & 4 \\
\hline $41 \mathrm{SY} 41$ & 1 \\
\hline $41 \mathrm{SY} 45$ & 8 \\
\hline 41SY92 & 10 \\
\hline $41 \mathrm{TT} 2$ & 36 \\
\hline $41 \mathrm{TT} 4$ & 89 \\
\hline $41 \mathrm{TT6}$ & 90 \\
\hline $41 \mathrm{TT} 7$ & 196 \\
\hline $41 \mathrm{TT} 11$ & 9 \\
\hline $41 \mathrm{TT} 13$ & 54 \\
\hline $41 \mathrm{TT} 17$ & 14 \\
\hline 41TT18 & 77 \\
\hline $41 \mathrm{TT} 36$ & 4 \\
\hline $41 \mathrm{TT} 110$ & 17 \\
\hline $41 \mathrm{TT} 111$ & 9 \\
\hline $41 \mathrm{TT} 135$ & 1 \\
\hline 41TT321 & 3 \\
\hline 41TT550 & 89 \\
\hline 41TT650 & 3 \\
\hline $41 \mathrm{TT} 653$ & 12 \\
\hline $41 \mathrm{TT} 718$ & 6 \\
\hline $41 \mathrm{TT} 741$ & 15 \\
\hline $41 \mathrm{TT} 851$ & 2 \\
\hline $41 \mathrm{TT} 852$ & 6 \\
\hline $41 \mathrm{TT} 853$ & 8 \\
\hline Raymond Powell & 6 \\
\hline Piney Creek & 26 \\
\hline Dellwood Branch & 20 \\
\hline 41UR1 & 37 \\
\hline 41UR2 & 140 \\
\hline
\end{tabular}


Table 1. East Texas sites/collections with Caddo ceramic vessels, cont.

\begin{tabular}{|c|c|}
\hline Site (with trinomial) or Collection (by County) & No. of vessels in database \\
\hline 41UR3 & 5 \\
\hline 41UR5 & 15 \\
\hline 41UR7 & 3 \\
\hline 41UR10 & 1 \\
\hline 41UR17 & 22 \\
\hline 41UR18 & 14 \\
\hline 41UR21 & 33 \\
\hline 41UR30 & 120 \\
\hline 41UR105 & 2 \\
\hline 41UR106 & 1 \\
\hline 41UR109 & 8 \\
\hline 41UR133 & 12 \\
\hline 41UR315 & 236 \\
\hline 41UR317 & 88 \\
\hline 41UR318 & 43 \\
\hline 41UR319 & 13 \\
\hline 41UR320 & 44 \\
\hline 41UR326 & 72 \\
\hline 41UR327 & 1 \\
\hline A. Davis & 7 \\
\hline Keeling & 34 \\
\hline Pine Bluff & 1 \\
\hline Glasco & 1 \\
\hline Upshur Co. & 1 \\
\hline Boyce Smith Collection (Upshur Co.) & 10 \\
\hline $41 \mathrm{VN} 3$ & 1 \\
\hline $41 \mathrm{VN} 6$ & 9 \\
\hline 41WD1 & 2 \\
\hline $41 \mathrm{WD} 2$ & 26 \\
\hline 41WD4 & 17 \\
\hline 41WD5 & 2 \\
\hline 41WD14 & 2 \\
\hline 41WD30 & 1 \\
\hline 41WD31 & 2 \\
\hline 41WD33 & 4 \\
\hline 41WD52 & 9 \\
\hline 41WD57 & 1 \\
\hline 41WD60 & 59 \\
\hline 41WD108 & 4 \\
\hline 41WD109 & 46 \\
\hline 41WD117 & 1 \\
\hline 41WD208 & 16 \\
\hline $41 \mathrm{WD} 244$ & 9 \\
\hline $41 \mathrm{WD} 354$ & 5 \\
\hline
\end{tabular}


Table 1. East Texas sites/collections with Caddo ceramic vessels, cont.

\begin{tabular}{ll}
\hline Site (with trinomial) or Collection (by County) & No. of vessels in database \\
\hline 41 WD371 & 5 \\
41 WD382 & 1 \\
41 WD482 & 1 \\
41 WD503 & 7 \\
41 WD507 & 1 \\
41 WD538 & 9 \\
41 WD586 & 5 \\
41 WD589 & 2 \\
Caney Creek & 58 \\
Wright & 1 \\
& \\
Arms Creek (Marion-Upshur Co.) & 17 \\
Clay Ball (Upper Neches) & 9 \\
Buddy Jones Collection-East Texas & 5 \\
Titus phase series & 219 \\
Upper Neches series & 8 \\
Upper Neches series, Buddy Jones Collection & 33 \\
Brooklyn Museum & 3 \\
Stock Pond (Big Cypress basin) & 25 \\
TPWD & 3 \\
\hline Total number of vessels in the database & 8068 \\
\hline
\end{tabular}

Table 2. Louisiana sites/collections with Caddo ceramic vessels.

\begin{tabular}{lc}
\hline Site (with trinomial) or Collection (by Parish) & No. of vessels in database \\
\hline 16BO236 & 9 \\
$16 \mathrm{BO} 237$ & 1 \\
16CD12 & 16 \\
16CD13 & 190 \\
16DS4 & \\
16DS212 & 19 \\
16NA9 & 1 \\
16NA13 & 2 \\
16NA14 & 1 \\
16NA16 & 1 \\
16RR1 & 2 \\
16SA1 & 5 \\
16SA30, B & \\
\hline
\end{tabular}


Table 2. Louisiana sites/collections with Caddo ceramic vessels, cont.

\begin{tabular}{lc}
\hline Site (with trinomial) or Collection (by Parish) & No. of vessels in database \\
\hline 16SA37a & 22 \\
16SA67 & 1 \\
16SA91 & 1 \\
X16SA4* (also from 16SA30, B) & 1 \\
DeSoto Parish & 1 \\
C. C. Jones & 3 \\
\hline Total number of vessels in the database & 357 \\
\hline
\end{tabular}

*SMU trinomial with the $\mathrm{X}$ prefix

Table 3. Oklahoma sites/collections with Caddo ceramic vessels.

\begin{tabular}{ll}
\hline Site (with trinomial) or Collection (by County) & No. of vessels in database \\
\hline $34 \mathrm{CH} 1$ & 3 \\
$34 \mathrm{CH} 112$ & 10 \\
$34 \mathrm{MC} 1$ & 36 \\
$34 \mathrm{MC} 6$ & 19 \\
$34 \mathrm{MC} 8$ & 3 \\
$34 \mathrm{MC} 9$ & 1 \\
$34 \mathrm{MC} 11$ & 66 \\
$34 \mathrm{MC} 12$ & 7 \\
$34 \mathrm{MC} 54$ & 20 \\
$34 \mathrm{MC} 84$ & 3 \\
$34 \mathrm{MC} 105$ & 3 \\
$34 \mathrm{MC} 215$ & 110 \\
\hline Total number of vessels in database & 281 \\
\hline
\end{tabular}

Table 4. Arkansas sites/collections with Caddo ceramic vessels.

\begin{tabular}{ll}
\hline Site (with trinomial) or Collection (by County) & No. of vessels in database \\
\hline 3LA1 & 163 \\
3LA27 & 33 \\
3LA28 & 4 \\
3LA38 & 8 \\
3LA48 & 46 \\
3LA84 & 5 \\
3LA97 & 67
\end{tabular}


134 Journal of Northeast Texas Archaeology 71 (2017)

Table 4. Arkansas sites/collections with Caddo ceramic vessels, cont.

\begin{tabular}{lc}
\hline Site (with trinomial) or Collection (by County) & No. of vessels in database \\
\hline 3MI1 & 21 \\
3MI6 & 252 \\
3MI29 & 8 \\
\hline Total number of vessels in database & 607 \\
\hline
\end{tabular}

\section{Acknowledgments}

The efforts of Bo Nelson and Mark Walters in the Caddo vessel documentation effort over the last decade and more are too numerous to mention. Thanks to Jeff Girard and Duncan McKinnon for sharing ceramic vessel database information, and I appreciate Ross Fields sharing the PDF of the 41MR63 technical report. Thanks also to Julian A. Sitters who assisted with the documentation of the final round of vessels at the Texas Archeological Research Laboratory at The University of Texas at Austin.

\section{References Cited}

McKinnon, D. P.

2016 Distribution of Design: The Rayed Circle. Caddo Archeology Journal 26:29-42. 